\title{
Association of Chemokine (C-C Motif) Receptor 5 and Ligand 5 with Recovery from Major Depressive Disorder and Related Neurocognitive Impairment
}

\author{
Olivia Bauer ${ }^{a}$ Vladimir M. Milenkovic ${ }^{a}$ Sven Hilbert $^{b} \quad$ Nina Sarubin ${ }^{c, d}$ \\ Johannes Weigl ${ }^{a} \quad$ Lisa-Marie Bahr ${ }^{a}$ Thomas C. Wetter ${ }^{a}$ Barbara Heckel $^{a}$ \\ Christian H. Wetzel ${ }^{a}$ Rainer Rupprecht ${ }^{a} \quad$ Caroline Nothdurfter $^{a}$ \\ aDepartment of Psychiatry and Psychotherapy, University of Regensburg, Regensburg, Germany; ${ }^{b}$ Faculty of \\ Human Sciences, University of Regensburg, Regensburg, Germany; ${ }^{C}$ Hochschule Fresenius, University of Applied

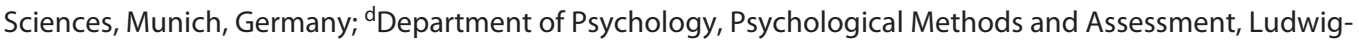 \\ Maximilians-Universität, Munich, Germany
}

\section{Keywords}

Chemokine ( $\mathrm{C}-\mathrm{C}$ motif) receptor $5 \cdot$ Chemokine (C-C motif) ligand 5/RANTES · Major depressive disorder · Cognitive impairment · Cambridge Neuropsychological Test Automated Battery

\begin{abstract}
Introduction: Inflammatory processes play an important role in the pathophysiology of major depressive disorder (MDD), but their relevance for specific symptoms such as neurocognitive impairment is rarely investigated. Methods: In this observational study, we investigated the changes of leukocyte chemokine (C-C motif) receptor 5 (CCR5) and ligand 5 (CCL5) mRNA levels and inflammatory cytokines in 60 MDD patients before (PRE) and after 5 weeks (W5) of antidepressive treatment in relation to therapy response and alterations in cognitive functions by means of the Cambridge Neuropsychological Test Automated Battery (CANTAB). We hypothesized that elevated CCR5 and CCL5 levels in depressed patients would decrease upon treatment and could differ with regard to cognitive impairment associated with
\end{abstract}

Olivia Bauer and Vladimir M. Milenkovic contributed equally to this work.

karger@karger.com www.karger.com/nim

Karger $\stackrel{\text { ' }}{5}$

GOPEN ACCESS
C 2021 The Author(s).

Published by S. Karger AG, Basel

This is an Open Access article licensed under the Creative Commons Attribution-NonCommercial-4.0 International License (CC BY-NC) (http://www.karger.com/Services/OpenAccessLicense), applicable to the online version of the article only. Usage and distribution for commercial purposes requires written permission.
MDD. Results: Both CCR5 and CCL5 levels were significantly decreased in the responder group compared to nonresponders even before treatment. The cytokine IL- 6 as a marker of inflammation in depression did not show a difference before treatment in future responders versus nonresponders, but decreased significantly upon antidepressive therapy. Regarding neurocognitive impairment in MDD patients, an increased misperception of the emotion "anger" after 5 weeks of treatment proved to be associated with a more pronounced change in CCR5, and the perception of the emotion "disgust" became faster along with a stronger decrease in CCL5 over the same time. Executive functions typically impaired in MDD patients were not markedly associated with alterations in CCR5/CCL5. Discussion: CCR5 and CCL5 are important in the targeting of immune cells by HIV. This is the first study providing valuable hints that both CCR5 and CCL5 might also serve as markers of therapy response prediction in MDD. Regarding neurocognitive impairment in depression, CCR5 and CCL5 did not reveal characteristic changes upon MDD treatment such as executive functions, which are probably delayed. However, changes of emotional perception appear to be an earlier responding feature.

(c) 2021 The Author(s).

Published by S. Karger AG, Basel

Caroline Nothdurfter

Department of Psychiatry and Psychotherapy, University of Regensburg

Universitätsstrasse 84

DE-93053, Regensburg (Germany)

caroline.nothdurfter@medbo.de 


\section{Introduction}

Major depressive disorder (MDD) is a highly prevalent and disabling disorder that is expected to become the largest global burden of disease within the next 2 decades according to the World Health Organization [1]. Pathophysiological mechanisms underlying depression are manifold and complex - among these are monoamine deficiency, abnormalities in the hypothalamic-pituitaryadrenal axis, or perturbations in neurogenesis [2]. There is accumulating evidence that suggests an association of inflammatory processes with MDD, thereby involving Creactive protein and inflammatory cytokines [3-5]. Recent data have further implicated a related family of immune proteins referred to as chemokines in many neuroimmune processes relevant to psychiatric disorders such as depression [6-9]. Chemokines are small (7-12 kDa) cytokines that induce directed chemotaxis and mediate leukocyte migration and inflammatory response [10]. Anti-inflammatory compounds therefore are and remain promising treatment approaches in MDD [11].

In general terms of therapy strategies for MDD, most treatments focus on the main symptoms such as depressed mood, loss of interest, and reduced energy. Cognitive dysfunction (such as reduced concentration and attention), however, very often remains insufficiently acknowledged. It is obvious that mechanisms underlying such neurocognitive impairment need to be investigated in more depth to optimize treatment strategies. In a study by Zhou et al. [12], cognitive impairment could be associated with specific alterations in chemokine levels. It was shown that decreasing the function of the chemokine (C-C motif) receptor 5 (CCR5) increases long-term potentiation and hippocampus-dependent memory in mice. Gates and colleagues [13] demonstrated similar effects of CCR5 suppression in humans - the use of CCR5 inhibitors could improve global neurocognitive functioning in patients suffering from HIV-associated neurocognitive disorder.

CCR5, also known as CD195, is a G protein-coupled chemokine receptor that is expressed on the surface of $\mathrm{T}$ cells, macrophages, dendritic cells, eosinophils, microglia, and certain cancer cells [14-16]. CCR5 plays an important role in HIV-1 infection because the virus uses CCR5 to enter the target immunological host cells $[17,18]$. In addition, other infectious diseases, such as malaria, involve CCR5-mediated recruitment of immune cells [19]. However, CCR5 is also involved in cancer biology [20,21] and brain pathologies, such as the inflammatory response in stroke [22]. A natural ligand of CCR5 is, among others, chemokine (C-C motif) ligand 5 (CCL5) - a chemotactic cytokine also known as RANTES (regulated on activation, normal $\mathrm{T}$ cell expressed and secreted) $[23,24]$. CCL5/ RANTES is produced by cytotoxic T cells and is likewise important in the defense against HIV infection by competitive binding to the receptor CCR5 $[25,26]$.

With regard to MDD, there is evidence that CCR5 as well as CCL5 levels are increased in patients suffering from depressive disorder compared to healthy subjects [27]. However, a time course of CCR5 development during treatment of MDD has not yet been shown. Furthermore, the role of CCR5 (and its ligand CCL5) in neurocognitive impairment in MDD, which is a very common and often underestimated symptom, still has to be clarified. Our study therefore aimed to investigate the change of CCR5 and CCL5 mRNA levels in leukocytes and neurocognitive impairment of 60 inpatients suffering from MDD before (PRE) and after 5 weeks (W5) of antidepressive treatment. Considering previous data of CCR5 and CCL5 in depression, we hypothesized that elevated CCR5 and CCL5 levels in MDD patients would decrease upon successful treatment. In addition, we expected improvements of cognitive impairment upon antidepressive therapy to be related to a decrease in CCR5 (and CCL5) levels.

\section{Subjects and Methods}

\section{Study Overview}

Seventy depressed inpatients, treated at the Department of Psychiatry and Psychotherapy, University of Regensburg, Germany, were included in this open-label 5-week observational study. Of these patients, 60 completed the study; 10 failed the final examination W5. The recruitment period lasted from August 2018 to February 2020. Since our study was designed to explore potential differences in clinical response independent of treatment, leukocyte CCR5 and CCL5 mRNA levels, inflammatory cytokines, and cognitive impairment in MDD, the following were defined as primary outcome measures: severity of depression assessed by Hamilton Depression Rating Scale (HAMD-21) sum scores [28], CCR5/ CCL5 mRNA levels, IL-1 $\beta /$ IL-6/TNF- $\alpha$, and cognitive impairment measured by means of Cambridge Neuropsychological Test Automated Battery (CANTAB) scores [29, 30]. Therapy response was defined as a reduction of at least $50 \%$ in the HAMD-21 sum score after 5 weeks of treatment (W5) compared to baseline (PRE). All raters were experienced psychiatrists and blind to CCR5/CCL5, cytokines, and CANTAB measurements. Raters were not changed in a single patient throughout the whole study period. Full blood samples for the assessment of CCR5/CCL5 mRNA levels in leukocytes $(2 \times 9.5 \mathrm{~mL}$ EDTA blood collection tubes $)$ and cytokines in plasma (9.5 $\mathrm{mL}$ LiHe tubes) were collected before antidepressant treatment started (PRE) and after 5 weeks of treatment (W5) as well as the CANTAB. Antidepressant medication was not controlled in this study, and it was chosen according to clinical judg- 
ment at the discretion of the psychiatrist in attendance. Table 1 gives an overview of the medication patients received.

The study was carried out according to the World Medical Association Declaration of Helsinki (http://www.wma.net) and had been approved by a local ethics committee (intramural review panel of the University Regensburg, Faculty of Medicine). Clinical trial number: Z-2018-1030-3; reference number of the ethics committee: 18-1037-101. The study has been registered at the German Clinical Trials Register (http://www.drks.de) under the number DRKS00023311.

\section{Eligibility}

Seventy inpatients (between 18 and 60 years of age) with an MDD episode according to DSM-IV criteria [31] were included in the study after the procedures had been fully explained and written informed consent had been obtained. Patients had to show clear signs of an MDD episode with a HAMD-21 sum score of at least 17. All participants were free of comorbid psychiatric disorders and acute somatic disorders; chronic somatic disorders such as arterial hypertension or hypothyroidism had to show normal values under stable respective medication. Regular intake of steroids or nonsteroidal anti-inflammatory drugs was not allowed and was controlled 3 days prior to and during the study. Female patients could not enter the study in case of pregnancy or breast feeding. Patients did not receive payment or other rewards for study participation. Sixty (27 males and 33 females) out of 70 patients completed the full study period up to week 5 including 2 blood withdrawals for CCR5/CCL5 determinations, 2 HAMD-21 ratings, and underwent CANTAB testing twice before (PRE) and after 5 weeks of treatment (W5), respectively. Clinical and demographic characteristics of all patients who completed the study are given in Table 2 .

\section{Isolation of PBMCs}

$2 \times 9.5 \mathrm{~mL}$ of peripheral blood was drawn by venipuncture into EDTA blood collection tubes for immediate use. 15-mL Pancoll human density 1.077 g/mL (PanBiotech, Aidenbach, Germany) was loaded into the upper chamber of a 50-mL LeucoSep tube (Greiner, Erlangen, Germany) to be centrifuged at $900 \mathrm{~g}$ for $1 \mathrm{~min}$ at room temperature (RT). Then, $12 \mathrm{~mL}$ of EDTA whole blood was added and centrifuged at $400 \mathrm{~g}$ for $15 \mathrm{~min}$ at RT. Afterwards, the peripheral blood mononuclear cell (PBMC) layer was collected and transferred to an ice-cold $50-\mathrm{mL}$ tube. It was filled to the maximum volume of $50 \mathrm{~mL}$ with $\mathrm{PBS} / 0.05 \%$ BSA precooled at $4^{\circ} \mathrm{C}$. Cells were washed twice with $\mathrm{PBS} / 0.05 \%$ BSA by centrifugation at

Table 1. Antidepressive medication

\begin{tabular}{lc}
\hline Drug & Sample, \% \\
\hline SSRI & 38.3 \\
SNRI & 40.0 \\
Tricyclic antidepressant & 6.7 \\
Mirtazapine & 16.7 \\
Agomelatine & 1.7 \\
Bupropion & 13.3 \\
Mood stabilizer & 6.7 \\
Atypical neuroleptic & 70.0 \\
\hline Drugs & \\
1 & 21.7 \\
2 & 58.3 \\
3 & 20.0 \\
\hline
\end{tabular}

In the left column of the upper part, drugs prescribed to patients are listed. The right column of the upper part represents the percentage of patients who received the respective drug. In the left column of the lower part, the number of drugs prescribed in parallel is listed. The right column of the lower part shows the percentage of patients who received such combinations. SSRI, selective serotonin reuptake inhibitors; SNRI, serotonin noradrenaline reuptake inhibitors.

Table 2. Clinical and demographic data of depressive patients

\begin{tabular}{|c|c|c|c|c|c|c|}
\hline \multirow{2}{*}{ Sex (male/female) } & \multirow{2}{*}{$\begin{array}{l}\begin{array}{l}\text { All patients } \\
(n=60)\end{array} \\
27 / 33\end{array}$} & \multirow{2}{*}{$\begin{array}{l}\begin{array}{l}\text { Responder } \\
(n=32)\end{array} \\
14 / 18\end{array}$} & \multirow{2}{*}{$\begin{array}{l}\text { Nonresponder } \\
(n=28)\end{array}$} & \multicolumn{3}{|c|}{ Statistical evaluation } \\
\hline & & & & & & \\
\hline$t$ test & mean $\pm \mathrm{SD}$ & mean $\pm S D$ & mean $\pm S D$ & $t$ & df & $p$ value \\
\hline HAMD-21, PRE & $24,417 \pm 5,652$ & $24,844 \pm 5,600$ & $23,929 \pm 5,773$ & -0.621 & 56.441 & 0.537 \\
\hline HAMD-21, W5 & $12,533 \pm 6,917$ & $7,563 \pm 3,426$ & $18,214 \pm 5,322$ & 9.073 & 44.981 & $<0.001^{*}$ \\
\hline Age, years & $38,167 \pm 12,933$ & $40,313 \pm 12,540$ & $357,143 \pm 13,163$ & -1.380 & 56.099 & 0.173 \\
\hline Age of onset, years & $32,088 \pm 13,072$ & $34,065 \pm 13,446$ & $29,731 \pm 12,457$ & -1.262 & 54.424 & 0.213 \\
\hline Depressive episodes, $n$ & $3,449 \pm 3,446$ & $3,393 \pm 2,726$ & $3,524 \pm 4,297$ & 0.122 & 31.756 & 0.903 \\
\hline Duration of index episode, weeks & $34,550 \pm 76,160$ & $32,688 \pm 91,024$ & $36,679 \pm 56,153$ & 0.207 & 52.441 & 0.837 \\
\hline
\end{tabular}

Data represent mean \pm SD (standard deviation). Statistical parameters of Welch sample $t$ tests for unequal variances are provided. Significant results are printed in bold and marked with an asterisk. Clinical response (responders) was defined by a reduction of at least $50 \%$ of the PRE HAMD-21 score after 5 weeks of treatment. HAMD-21, Hamilton Depression Rating Scale, 21-item version; PRE, time at admission; W5, after 5 weeks of treatment. * Significant at $\alpha=0.05$. 
$250 \mathrm{~g}$ for $10 \mathrm{~min}$ at $4^{\circ} \mathrm{C}$, respectively. Cell number was counted and extrapolated to $1 \mathrm{~mL}$ of patient's blood before freezing cells at $-80^{\circ} \mathrm{C}$ for further use.

PCR Analyses of Leukocyte CCR5 and CCL5 Levels

To determine the expression of CCR 5 and CCL5 in leukocytes, RNA was extracted from 2 million leukocytes using RNA Plus Kit (Macherey-Nagel, Düren, Germany) according to the manufacturer's instructions. First-strand cDNA synthesis from $500 \mathrm{ng}$ of total RNA was performed with QuantiTect Reverse Transcription Kit (Qiagen, Hilden, Germany). Quantitative realtime PCR experiments were performed with the Rotor-Gene-Q device (Qiagen) using the $1 \times$ Takyon SYBR Master Mix (Eurogentec, Köln, Germany) and specific intron spanning primers, listed in Table 3. Measurements were performed in triplicate, and results were analyzed with Rotor-Gene-Q software version 2.3 (Qiagen) applying the $\Delta \Delta \mathrm{Ct}$ method for relative quantification.

\section{CCR5-Delta32 Mutation}

Genomic DNA was extracted from 2 million PBMCs with the QIAamp DNA mini kit (Qiagen) according to the manufacturer's protocol. DNA quality was assessed utilizing optical absorbance and gel electrophoresis. The portion of the exon 2 of the CCR5 gene containing the delta 32 mutation was PCR amplified and sequenced using the Sanger method. Sequencing primers used in this study are listed in Table 3. Sequencing data were analyzed using the SnapGene software (from GSL Biotech; available at snapgene.com).

\section{V-PLEX Immunoassay}

Plasma levels of the cytokines IL-1 $\beta$, IL- 6 , and TNF- $\alpha$ were measured by means of the V-PLEX Cytokine Panel 1 (Meso Scale Discovery, Rockville, MD, USA), according to the manufacturer's instructions. For that purpose, $50 \mu \mathrm{L}$ of each 1:2 diluted plasma sample was added in duplicate to each well of the precoated 96-well plate and incubated at RT for $2 \mathrm{~h}$ under continuous shaking. After washing plates 3 times with wash buffer (Meso Scale Discovery, Rockville, MD, USA), sulfotag detection antibody cocktail (Meso Scale Discovery) was added to each well. Plates were incubated for $2 \mathrm{~h}$ at RT under continuous shaking. In the end, plates were washed again, and data were acquired on the Meso QuickPlex SQ 120 electrochemiluminescence plate scanner after addition of $150 \mu \mathrm{L}$ of 2 $\times$ read buffer. Data were analyzed using the Discovery Workbench 4.0 software (Meso Scale Discovery). Standard curves for each cytokine were generated using serial 4 -fold dilutions of the standards provided in the kit. Cytokine concentrations were determined from the standard curve using a four-parameter logistic fit curve to convert the mean light intensities into concentrations. The lower and upper limits of quantification were determined for each cytokine and for each plate.

\section{Cambridge Neuropsychological Test Automated Battery (CANTAB)}

Depressive patients performed the Cambridge Neurocognitive Test Automated Battery (CANTAB - http://www.cantab.com), which is a validated and standardized tool for the assessment of neurocognitive functioning $[29,30]$. The subset for affective disorders consists of 5 subtests.

\section{Rapid Visual Information Processing}

A white box is shown in which digits from 2 to 9 appear in a pseudo-random order at a rate of 100 digits per minute. Patients are requested to detect target sequences of digits (e.g., 3-5-7) and respond by pressing a button as quickly as possible.

\section{Spatial Working Memory}

This test requires retention and manipulation of visuospatial information and provides a measure of strategy as well as working memory errors. Patients are presented a number of boxes on the screen. By selecting boxes and using a process of elimination, patients should find 1 yellow "token" in each of the number of boxes and use them to fill up an empty column on the side of the screen.

\section{Delayed Match to Sample}

Delayed match to sample assesses both simultaneous visual matching ability and short-term visual recognition memory, for nonverbalizable patterns. Patients are presented a complex visual pattern that is both abstract and nonverbal, followed by 4 similar patterns after a brief delay. They have to select the pattern, which exactly matches the sample. In some trials, the sample and the choice patterns are shown simultaneously, and in others, there is a delay before the 4 choices appear.

\section{One Touch Stockings of Cambridge}

This is a test of executive function, based upon the Tower of Hanoi test, which assesses both the spatial planning and the working memory subdomains. Patients are shown 2 displays containing 3 colored balls. These displays are presented in such a way that they can be easily perceived as stacks of colored balls held in stockings suspended from a beam. Patients are requested to copy a given pattern by moving only 1 ball at a time.

Table 3. Primers used for genotyping and qRT-PCR

\begin{tabular}{lll}
\hline Primer name & Forward primer $\left(5^{\prime}-3^{\prime}\right)$ & Reverse primer $\left(5^{\prime}-3^{\prime}\right)$ \\
\hline CCR5 $\Delta 32$ & TTAAAAGCCAGGACGGTCAC & GACCAGCCCCAAGATGACTA \\
CCR5 & GAGACATCCGTTCCCCTACA & GGCAGGGCTCCGATGTATAA \\
CCL5 & TACACCAGTGGCAAGTGCTC & TGTACTCCCGAACCCATTTC \\
HPRT1 & TTGCTTTCCTTGGTCAGGCA & ATCCAACACTTCGTGGGGTC \\
\hline
\end{tabular}

CCR5, chemokine (C-C motif) receptor 5; CCL5, chemokine (C-C motif) ligand 5; HPRT1, hypoxanthine phosphoribosyltransferase 1 housekeeping gene.

Association of CCR5 and CCL5 with

MDD and Cognitive Impairment
Neuroimmunomodulation 2020;27:152-162 DOI: $10.1159 / 000513093$ 


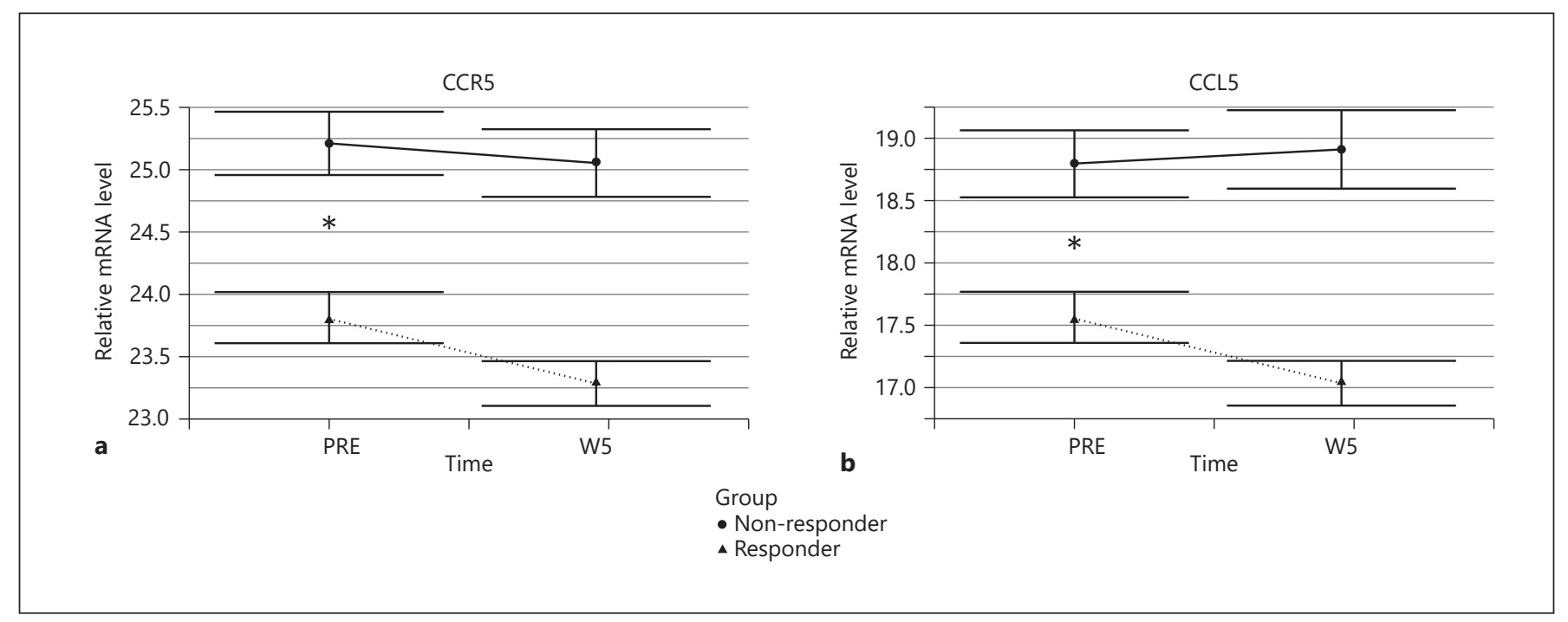

Fig. 1. CCR5 and CCL5 levels in relation to clinical response. Relative mRNA levels of CCR5 (a) and CCL5 (b) measured by real-time PCR in leukocytes of depressed patients in relation to clinical response after 5 weeks of treatment. Clinical response (responder vs. nonresponder) was defined by a reduction of at least $50 \%$ of the PRE (at admission) HAMD-21 score after 5 weeks of treatment (W5). Significant differences are marked with an asterisk. CCR5, chemokine (C-C motif) receptor 5; CCL5, chemokine (C-C motif) ligand 5.

Emotion Recognition Task

Emotion recognition task (ERT) measures the ability to identify 6 basic emotions (sadness, happiness, fear, anger, disgust, or surprise) in facial expressions along a continuum of expression magnitude. Computer-morphed images derived from real individuals, each showing a specific emotion, are displayed for 200 $\mathrm{ms}$. The CANTAB is computerized and was carried out twice in every subject, at PRE (baseline) and W5 (5 weeks after treatment).

\section{Statistical Analyses}

All analyses were conducted using the statistical software $\mathrm{R}$ [32]. Alterations in the levels of CCR5, CCL5, and cytokines over time (PRE, before antidepressive therapy; W5, after 5 weeks of treatment) for responders and nonresponders were analyzed using linear mixed regression models using the package "lme4" [33]. Type I error probabilities for the regression coefficients were calculated using the package "lmerTest" [34]. Due to triplicate determination of CCR5/CCL5 and duplicate cytokine levels, the arithmetic mean of each value per single sample was determined and used as a dependent variable. Unconditional random effects models (UREMs) were estimated to analyze the effect of possible within-subject variances of CCR5/CCL5 levels and cytokine concentrations. Subsequently, intraclass correlations (ICCs) were calculated to quantify the inter- and innersubject variation. For the analysis change in CCR5/CCL5 levels and cytokine concentrations, linear growth variables "time" were created, using the coding 0 and 1 for the time points PRE and W5, respectively. Dummy coding (nonresponder: 0; responder: 1) was applied to create the variable "response" for the estimation of the relationship of treatment response (according to HAMD-21 at W5) on the mean CCR5/CCL5 levels and cyto- kine concentrations. In order to analyze the relationship between treatment response and the change in CCR5/CCL5 levels over time points PRE and W5, an interaction term entered the analysis. The model was estimated, allowing for varying intercepts (i.e., initial CCR5/CCL5 levels and cytokine concentrations) between patients, thereby accounting for the nesting in the data within subjects. For a detailed overview of the interpretation of the effects of this type of dummy coding for longitudinal intervention studies analyzed with linear mixed models, see Hilbert et al. [35]. All tests were run two-tailed, and all type I error probabilities underwent 50 -fold Bonferroni correction to account for the total ten fitted regression models. The $p$ values are reported without correction, yet for that CANTAB models, due to the large amount of tests, statistical significance was only assumed for values of $p<0.001$.

\section{Results}

\section{Clinical Characteristics of Patients}

There were no marked differences between responders and nonresponders with regard to clinical or demographic variables (all $p>0.05$ for all comparisons; see Table 2). Regarding HAMD-21 treatment response (reduction of at least $50 \%$ in the HAMD-21 sum score after 5 weeks of medication), 32 patients were responsive to antidepressive treatment, while 28 patients were nonresponders (see Table 2). 
Table 4. CCR5 and CCL5 levels in relation to clinical response

\begin{tabular}{lclllc}
\hline Coefficient & Estimate & SE & df & $t$ & $p$ value \\
\hline CCR5 & & & & & \\
(intercept) & 25.211 & 0.334 & 80.110 & 75.481 & $<\mathbf{0 . 0 0 1}^{*}$ \\
Response & -1.408 & 0.457 & 80.110 & -3.078 & $\mathbf{0 . 0 0 3}^{*}$ \\
Time & -0.169 & 0.272 & 58.000 & -0.624 & 0.535 \\
Response:time & -0.352 & 0.372 & 58.000 & -0.947 & 0.347 \\
\hline CCL5 & & & & & \\
(intercept) & 18.785 & 0.352 & 85.987 & 53.387 & $<\mathbf{0 . 0 0 1}$ \\
Response & -1.229 & 0.482 & 85.987 & -2.550 & $\mathbf{0 . 0 1 3}$ \\
Time & 0.114 & 0.318 & 58.000 & 0.359 & 0.721 \\
Response:time & -0.633 & 0.436 & 58.000 & -1.452 & 0.152 \\
\hline
\end{tabular}

Relative mRNA levels of CCR5 (upper part) and CCL5 (lower part) and HAMD-21 scores were assessed at baseline (PRE) and after 5 weeks of treatment (W5). Clinical response (responder vs. nonresponder) was defined by a reduction of at least $50 \%$ of the PRE HAMD-21 score after 5 weeks of treatment. To analyze the relationship between treatment response and a change in CCR5/ CCL5 levels over time, an interaction term entered the analysis (response:time). Coefficient, regression coefficient; estimate, estimated parameter value; SE, standard error of the parameter estimate; df, degrees of freedom; $t, t$ value; $p$, probability of committing a type I error; CCR 5 , chemokine ( $\mathrm{C}-\mathrm{C}$ motif) receptor 5 ; CCL5, chemokine (C-C motif) ligand 5; intercept, intercept of the linear regression; time, dichotomous growth variable for the change between PRE and W5. Statistically significant effects $\left({ }^{*} \alpha=\right.$ 0.05 ) are highlighted in bold and are marked with an asterisk.

Analysis of Changes in CCR5 and CCL5 mRNA Levels

The UREMs estimated to compare the inter- and inner-subject variation resulted in ICCs of 0.685 for CCR 5 levels and 0.642 for CCL5 levels. This means that in both cases, $>60 \%$ of the variance was related to between-subject differences, and the application of mixed models, taking these differences into account, was clearly imperative. First, mixed regression models with dummy-coded variables "time" and "response" as well as their interaction term were estimated. As can be clearly seen in Table 4 and Figure 1a (CCR5) and Figure 1b (CCL5), the regression weights for "time" were significant and negative for both models, indicating a mean decrease for both CCR5 and CCL5 between time points PRE and W5. Except for the intercepts, no further regression weights turned out to be significant, so that the stronger decrease between responders and nonresponders remains a descriptive finding. Nonetheless, both mean CCR5 and CCL5 levels were significantly decreased in the responder group compared to nonresponders before treatment.

Association of CCR5 and CCL5 with

MDD and Cognitive Impairment
Table 5. Cytokine levels in relation to clinical response

\begin{tabular}{lrllrc}
\hline Coefficient & Estimate & SE & df & \multicolumn{1}{l}{$t$} & $p$ value \\
\hline$I L-1 \beta$ & & & & & \\
(intercept) & 0.076 & 0.167 & 111 & 0.454 & 0.651 \\
Response & 0.049 & 0.232 & 111 & 0.212 & 0.832 \\
Time & -0.004 & 0.236 & 111 & -0.017 & 0.987 \\
Response:time & 0.426 & 0.324 & 111 & 1.317 & 0.191 \\
\hline IL-6 & & & & & \\
(intercept) & 0.819 & 0.127 & 93.577 & 6.473 & $<\mathbf{0 . 0 0 1 *}$ \\
Response & -0.236 & 0.173 & 93.577 & -1.477 & 0.143 \\
Time & -0.300 & 0.128 & 58.000 & -2.345 & $\mathbf{0 . 0 2 3}$ \\
Response:time & 0.168 & 0.175 & 58.000 & 0.958 & 0.342 \\
\hline TNF- $\alpha$ & & & & & \\
(intercept) & 1.473 & 0.105 & 89.730 & 13.992 & $<\mathbf{0 . 0 0 1 *}$ \\
Response & 0.192 & 0.144 & 89.730 & 1.326 & 0.188 \\
Time & 0.035 & 0.101 & 58.000 & 0.350 & 0.727 \\
Response:time & -0.087 & 0.138 & 58.000 & -0.628 & 0.533 \\
\hline
\end{tabular}

Plasma concentrations of the cytokines IL-1 $\beta$, IL-6, and TNF- $\alpha$ and HAMD-21 scores were assessed at baseline (PRE) and after 5 weeks of treatment (W5). Clinical response (responder vs. nonresponder) was defined by a reduction of at least $50 \%$ of the PRE HAMD-21 score after 5 weeks of treatment. To analyze the relationship between treatment response and cytokine levels over time, an interaction term entered the analysis (response:time). Coefficient, regression coefficient; estimate, estimated parameter value; SE, standard error of the parameter estimate; df, degrees of freedom; $t, t$ value; $p$, probability of committing a type I error; intercept, intercept of the linear regression; time, dichotomous growth variable for the change between PRE and W5. Statistically significant effects $\left({ }^{*} \alpha=0.05\right)$ are highlighted in bold and are marked with an asterisk.

\section{Analysis of Changes in Cytokines $I L-1 \beta, I L-6$, and} TNF- $\alpha$ Levels

The UREMs for the cytokines showed ICCs of 0.462 for IL- 6 and 0.553 for TNF- $\alpha$ concentrations. The model for IL- $1 \beta$ showed a singular fit, and thus no ICC could be estimated, which may be due to $n=5$ missing values for this cytokine. Around $50 \%$ of the variance in the models is attributable to inter-subject differences in the cytokine concentration, except for IL- $1 \beta$, for which the relationship could not be determined due to the singular model fit. Mixed regression models were therefore estimated for all cytokines. Table 5 and Figure 2a (IL-6) and Figure 2b (TNF- $\alpha$ ) show that the only significant regression weight was the negative weight for the "time" variable of IL-6, that is, the mean IL- 6 concentration decreased significantly during the observed treatment period.

Neuroimmunomodulation 2020;27:152-162 


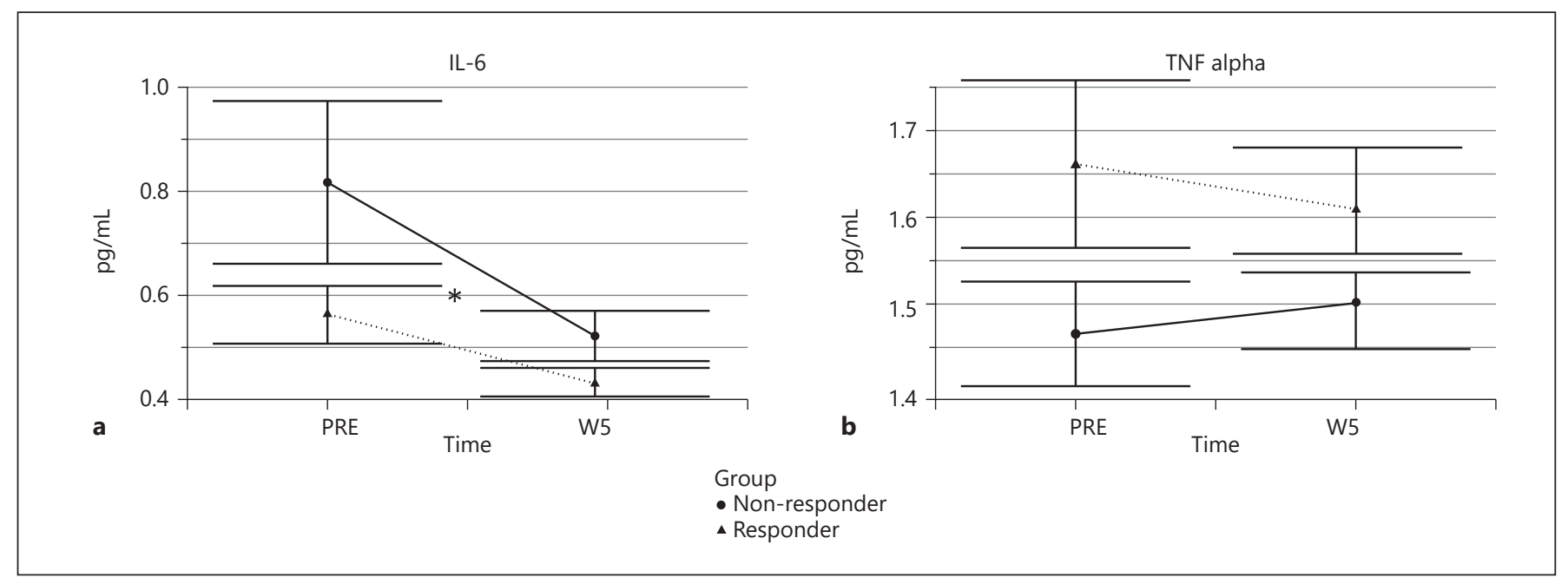

Fig. 2. Cytokine levels in relation to clinical response. Plasma concentrations of the cytokines IL-6 (a) and TNF- $\alpha$ (b) levels in relation to clinical response after 5 weeks of treatment were measured by means of a V-PLEX immunoassay. Clinical response (responder vs. nonresponder) was defined by a reduction of at least $50 \%$ of the PRE (at admission) HAMD-21 score after 5 weeks of treatment (W5). Significant differences are marked with an asterisk.

\section{Association of CANTAB Tasks with CCR5 and CCL5 Levels}

In a second step, the decrease in CCR5 and CCL5 levels was tested for associations with changes in CANTAB tasks. Instead of the dichotomous "group" variable, indicating the treatment response, the CANTAB parameters were entered in all models as covariables. Again, "time" as well as the interaction term between "time" and the covariable of the model was also modeled. As reflected by the interaction terms in Table 6, a change in the variable "ERTTFAA" (total false alarms "anger") between PRE and W5 was significantly increased along with a greater decrease in CCR5, and a change in the variable "ERTMDCRTD" (median correct reaction time "disgust") significantly decelerated along with a greater decrease in CCL5 over the same time. Implying values of $p<0.01$ as noteworthy descriptive tendencies, some One Touch Stockings of Cambridge (OTS) variables reflecting processing speed in executive function tasks (see legend to Table 6) also showed a trend towards an improvement associated with CCR5.

\section{CCR5-Delta32 Mutation}

Only 1 patient (in the nonresponder group) turned out to be homozygous for the CCR5 $\Delta 32$ mutation in our sample. We therefore consider the influence of this genetic variant negligible for treatment response, CCR5/ CCL5 levels, and cognitive impairment in the depressed patients of this sample.

\section{Discussion}

The aim of the present study was to assess CCR 5 and CCL5 mRNA levels in peripheral blood samples of depressed patients during antidepressive treatment, to correlate it with therapy success and neurocognitive impairment in MDD. This was an observational study that did not differentiate between specific pharmacological antidepressive treatments. We found CCR5 and CCL5 levels significantly decreased in the responder group compared to nonresponders before treatment. Descriptively, CCR5 showed a further decrease upon therapy, which was more pronounced in the responder group. CCL5 tended to decrease in the responder group, while it slightly increased in nonresponders upon treatment. Considering cytokine levels as representatives of inflammatory processes underlying MDD, IL-6 decreased significantly upon antidepressive therapy. However, this cytokine did not show a difference before treatment in future responders versus nonresponders. For TNF- $\alpha$, no differences before or upon treatment could be observed in this sample. Concerning neurocognitive impairment measured by means of the CANTAB, a misperception of the emotion "anger" significantly increased after 5 weeks of treatment in connection with the decrease of CCR5. The perception of the emotion "disgust" became faster along with a decrease in CCL5 over time. Executive functions typically impaired in MDD patients were not markedly associated with alterations in CCR5/CCL5. 
The development of CCR5 levels appears in line with our presumption that recovery from depression goes along with a return to lower levels of this chemokine receptor, which has already been described as increased during MDD compared to healthy subjects [27]. Nonetheless, further studies also show contradictory results regarding CCR5 in depression, and a very recent study investigating different chemokine receptors in bipolar patients detected decreased CCR5 expression compared to control [36]. However, this patient sample included depressed as well as manic patients, thereby suggesting a common pathophysiological concept with regard to chemokine receptors in bipolar disorder. In our study, bipolar patients were also included ( 4 out of 60 ), but the present state had to be depressed.

With regard to the ligand CCL5 and depressive disorder, the database is broader. There already exist studies observing the time course of this chemotactic cytokine during depression. Mindt et al. [37] found that patients treated with electroconvulsive therapy showed a decrease of CCL5 in cerebrospinal fluid of patients who responded to this intervention, which appears in line with the results of our study, although we just observed a tendency. Considering the comparison of depressed patients with healthy subjects, also for CCL5, results are contradictory in the literature. CCL5 was found to be decreased in serum of MDD patients with suicidal ideation compared to healthy controls by Grassi-Oliveira et al. [38]. However, a meta-analysis by Leighton and colleagues [39] including 73 studies could not confirm differences in blood or cerebrospinal fluid levels of CCL5 in depressed patients compared to healthy subjects.

A very recent study by Chen et al. [40] tried to investigate the role of CCR and CCL5 from a more interventional point of view: in a mouse model of circadian rhythm, intracerebral injection of CCL5 promoted a depressionlike behavior, which could be reversed by application of Met-RANTES, a CCR5 antagonist. These results support the hypothesis that a recovery from depression goes along with a decrease of CCR5 and CCL5 function/levels.

Although the decrease of CCR5 and CCL5 in our sample yet was just a tendency, it might have been statistically more reliable in a larger sample. In addition, future studies should choose considerably longer observation periods which might further sharpen this effect because inflammatory processes in depression might go far beyond clinically apparent symptoms [41]. Nonetheless, there was a marked difference at baseline, and future responders showed significantly lower CCR5 as well as CCL5 levels before therapy started, although baseline
Table 6. CANTAB values of depressed patients

\begin{tabular}{|c|c|c|c|c|c|}
\hline \multirow{2}{*}{$\begin{array}{l}\text { Coefficient } \\
\text { CCR5 }\end{array}$} & \multicolumn{2}{|c|}{ EstimateSE } & \multirow[t]{2}{*}{ df } & & \multirow[t]{2}{*}{$p$ value } \\
\hline & & & & & \\
\hline ercept) & 25.076 & 0.320 & 114.003 & 78.288 & $<0.001$ \\
\hline Time & -0.485 & 310 & 63.100 & 666 & 0.122 \\
\hline DTSMDLC6 & 0.000 & 0.000 & 8.626 & -2 & 0.004 \\
\hline Time:OTSMDLC6 & 0.000 & 0.000 & 66.826 & -0.202 & 0.841 \\
\hline (intercept) & 25.142 & 0.346 & 115.432 & 72.651 & $<0.001$ \\
\hline Time & -0 . & 0.320 & & & 0.099 \\
\hline $\mathrm{LC}$ & & 0 & & & 0.007 \\
\hline MLC6 & & 0 & & & 0.990 \\
\hline & 25 & 0.4 & 115.8 & 62 & $<0.001$ \\
\hline Tin & -0.4 & 0.388 & 6.915 & -1 & 0.245 \\
\hline OTSMLC & 0 & 0.000 & 92. & -2 & 0.005 \\
\hline TSMLC & & 0.000 & 63 & -0 & 0.718 \\
\hline & 24 & 0. & $10 \varepsilon$ & & $<0.001$ \\
\hline Tin & -1.1 & 0.286 & 5 & -4 & $<0.001$ \\
\hline ER & -0.0 & 0.045 & 112. & -1.2 & 0.209 \\
\hline Time:ERTTFAA & 0.156 & 0.042 & 59.884 & 3.684 & $<0.001^{*}$ \\
\hline & & & & & \\
\hline (n) & 17.6 & 0.43 & & 11 & $<0.001$ \\
\hline Time & 1813 & 0.613 & 3 & 2.957 & 0.004 \\
\hline ERTMDCRT & 0.000 & 0.000 & 92.954 & 1.429 & 0.156 \\
\hline Time:ERTMDCRTD & -0.001 & 0.000 & 72.179 & -3.445 & $<0.001^{*}$ \\
\hline (intercept) & 17.623 & 0.510 & 114.413 & 34.528 & $<0.001$ \\
\hline Time & 1.286 & 0.563 & 63.106 & 2.285 & 0.026 \\
\hline ERTM & & 0.000 & & & 0.253 \\
\hline Time:ERTMDRTD & -0.001 & 0.000 & 66.398 & -2.883 & 0.005 \\
\hline
\end{tabular}

Changes in CCR5 (upper part) and CCL5 (lower part) levels were tested for associations with changes in CANTAB tasks. The CambridgeNeuropsychological Test Automated Battery(CANTAB) was performed at admission (PRE) and after 5 weeks of treatment (W5) in parallel with CCR5/CCL5 measurements. Coefficient, regression coefficient; estimate, estimated parameter value; SE, standard error of the parameter estimate; df, degrees of freedom; $t$, $t$ value; $p$, probability of committing a type I error; CCR5, chemokine (C-C motif) receptor 5; CCL5, chemokine (C-C motif) ligand 5; intercept, intercept of the linear regression; time, dichotomous growth variable for the change between PRE and W5. For the CANTAB models, due to the large amount of tests, only values of $p<0.001$ were considered as statistically significant. Values of $p<$ 0.01 are listed as descriptive tendencies. OTS, One Touch Stockings of Cambridge; ERT, emotion recognition task. OTSMDLC6: OTS median latency to correct 6 moves, the median latency measured from the appearance of the stocking balls until the correct box choice was made by the subject when 6 moves was the solution. OTSMLC6: OTS mean latency to correct 6 moves, the mean latency measured from the appearance of the stocking balls until the correct box choice was made by the subject when 6 moves was the solution. OTSMLC: OTS mean latency to correct, the mean latency measured from the appearance of the stocking balls until the correct box choice was made by the subject. ERTTFAA: ERT total false alarms "anger," the total number of times the subject incorrectly selected the emotion "anger" across all assessed trials. ERTMDCRTD: ERT median correct reaction time "disgust," the median latency for a subject to correctly select the emotion "disgust" after being presented with a face stimulus showing disgust. ERTMDRTD: ERT median reaction time "disgust," the median latency for a subject to select the emotion word "disgust" after being presented with a face stimulus. 
HAMD-21 sum scores were quite equal in both groups. This maybe offers the opportunity of a therapy response prediction by means of this chemokine receptor and its ligand. This information might be of help for therapeutic decision-making in such way that patients rather predicted as nonresponders need earlier escalation of therapy, such as combination of antidepressants or electroconvulsive therapy.

One has to be aware that different antidepressive medication in this patient sample might be a potential confounder. However, there do not exist much data on the influence of such compounds on CCR5, and only 1 study investigated the effect of the SSRI citalopram on different chemokine receptors on PBMCs - CCR5 levels were not altered by citalopram treatment in the cell culture [42]. The tricyclic antidepressant desipramine has been shown to suppress central CCL5 overproduction in a mouse model of experimental autoimmune encephalomyelitis [43]. In our sample, no patient received this compound. More relevant in this context appears a study by Vollmar and colleagues [44] showing that the SNRI venlafaxine can suppress CCL5 production in inflamed CNS tissue in murine experimental autoimmune encephalomyelitis. In this sample, 9 out of 60 patients received venlafaxine. In addition, the SSRI fluoxetine decreased CCL5 production in an in vitro model of septic shock [45]. In summary, the effects of commonly prescribed antidepressants on CCL5 levels must not be underestimated. Future studies investigating the effect of CCR5 and CCL5 on depression should therefore control for antidepressive medication by comparing single compounds.

To complete the picture of inflammatory processes underlying the pathophysiology of depression, we measured specific cytokine levels before and after antidepressive treatment. Especially, IL- 6 is a well-documented marker that is elevated in depressed patients compared to healthy subjects $[46,47]$ and which decreases upon antidepressive treatment [48]. Very well in line with this broad database, IL-6 also decreased significantly in our sample upon therapy. To our knowledge, this is the first study investigating both IL-6 and CCR5/CCL5 in a time course of treatment, thereby offering the chance to distinguish between future clinical responders and nonresponders. Interestingly, there was no difference between baseline IL- 6 levels between these 2 groups, which is in contrast to the more differential picture of CCR5/CCL5 levels being lower in the responder group already before therapy. This dissociation of inflammatory markers appears to further strengthen the hypothesis that CCR5/ CCL5 might be interesting candidates for predictors of future antidepressive therapy response. With regard to TNF- $\alpha$, we did not find marked changes before or after antidepressive treatment, which is also in line with previous studies showing not quite consistent results comparing MDD patients with healthy controls [46] and the development of this cytokine along with antidepressive treatment [48-50]. To postulate an association of TNF- $\alpha$ with CCR5/CCL5 therefore does not appear appropriate for this study.

CCR5 is already known to be important for the entry of HIV into the host cell, and the homozygous $\Delta 32$ mutation in this gene prevents CCR5 cell-surface expression and thus lends a certain resistance to infection with this virus [51]. With probably $<1 \%$ prevalence, it is not very widespread $[52,53]$, which is in line with just 1 patient we discovered in our sample. So far, there is no evidence in the literature that the $\Delta 32$ mutation is relevant for the pathophysiology of MDD and related cognitive impairment. Very large samples would be necessary to answer this question, which we could not provide in this investigation.

With regard to CANTAB results in MDD patients in our sample, a misperception of the emotion "anger" significantly increased after 5 weeks of antidepressive treatment in connection with the decrease of CCR5, and the perception of the emotion "disgust" became faster over the same time in the ERT of the CANTAB along with a stronger decrease in CCL5. These results could be interpreted as a kind of sharpening of the perception of negative emotions going along with a downregulation of CCR5/CCL5 under antidepressive treatment. Furthermore, several OTS tasks representing executive functions such as spatial planning and working memory showed an improvement of processing speed over time associated with CCR 5 decreased strength. Improvement of OTS tasks is very well in line with what can be expected upon successful treatment of depression [54, 55]; however, these results should be just descriptively interpreted in our study. This is because the large number of statistical tests leads to a conservative correction of the type 1 error probability. Statistical significance was therefore interpreted only very cautiously. In addition, it might be assumed that changes of emotional perception show an earlier response compared to a delayed recovery of executive functions in depressed patients in relation to CCR5/CCL5 levels. The recovery from such typical neurocognitive deficits would possibly have been more marked after a longer follow-up period; they can outlast the remission from characteristic MDD symptoms such as depressed mood and loss of interest for quite some time [54]. Interestingly, a very recent study 
by Ozcan et al. [56] also showed an early improvement in emotion recognition in the CANTAB, while cognitive functions such as cognitive flexibility did not markedly improve in patients suffering from treatment-resistant MDD treated with repetitive transcranial magnetic stimulation over 4-6 weeks, similar to our study. For a differentiation of the responder versus nonresponder in cognitive functions along with CCR5/CCL5 levels, the sample size was too small in our study. However, one can postulate a certain recovery from depressive symptoms also in the group of nonresponders; they just did not reach the predefined level of $50 \%$ improvement in HAMD-21 scores.

In summary, the present study provides first hints for CCR 5 and CCL 5 as putative predictors of future therapy response in major depression. However, further studies with larger sample sizes, a longer observation period, and controlling for antidepressive medication are needed to confirm these results. Regarding the results of the CAN$\mathrm{TAB}$, we did not find strong evidence for a relevant relation between CCR5/CCL5 and a recovery from MDDassociated cognitive impairment after a 5-week treatment. However, changes of emotional perception appeared to respond earlier to antidepressive therapy.

\section{Acknowledgements}

We thank Professor Stefan Holdenrieder (Deutsches Herzzentrum München, Germany) for technical support with the cytokine ELISAs and Doris Melchner, Anett Dörfelt, and Stefanie Bader for valuable technical assistance.

\section{Statement of Ethics}

The study was carried out according to the World Medical Association Declaration of Helsinki (http://www.wma.net) and had been approved by a local ethics committee (intramural review panel of the University Regensburg, Faculty of Medicine). Clinical trial number: Z-2018-1030-3; reference number of the ethics committee: 18-1037101. The study has been registered at the German Clinical Trials Register (http://www.drks.de) under the number DRKS00023311. All subjects have given their written informed consent.

\section{Conflict of Interest Statement}

The authors report no biomedical financial interests or potential conflicts of interest.

\section{Funding Sources}

The study was funded by the Department of Psychiatry and Psychotherapy, University of Regensburg, Regensburg, Germany.

\section{Author Contributions}

O.B.: coordination, patient recruitment, CANTAB, blood sampling, PCR analyses, and preparation/writing of the manuscript. V.M.M. and C.H.W.: PCR analyses and preparation/writing of the manuscript. S.H.: statistical analyses and preparation/writing of the manuscript. N.S.: study design and statistical analyses. J.W., T.C.W., and B.H.: patient recruitment, HAMD-21 rating, and blood sampling. L.M.B.: study design, CANTAB, and PCR analyses. R.R.: study design, proposal, and preparation/writing of the manuscript. C.N.: study design, proposal, coordination, patient recruitment, HAMD-21 rating, blood sampling, and preparation/ writing of the manuscript.

\section{References}

1 Global Burden of Disease Study C. Global, regional, and national incidence, prevalence, and years lived with disability for 301 acute and chronic diseases and injuries in 188 countries, 1990-2013: a systematic analysis for the Global Burden of Disease Study 2013. Lancet. 2015;386: 743-800.

2 Belmaker RH, Agam G. Major depressive disorder. N Engl J Med. 2008 Jan 3;358(1):55-68.

3 Miller AH, Maletic V, Raison CL. Inflammation and its discontents: the role of cytokines in the pathophysiology of major depression. Biol Psychiatry. 2009 May 1;65(9):732-41.

4 Dowlati Y, Herrmann N, Swardfager W, Liu H, Sham L, Reim EK, et al. A meta-analysis of cytokines in major depression. Biol Psychiatry. 2010 Mar 1;67(5):446-57.

5 Haapakoski R, Mathieu J, Ebmeier KP, Alenius $\mathrm{H}$, Kivimäki M. Cumulative meta-analysis of interleukins 6 and $1 \beta$, tumour necrosis factor $\alpha$ and C-reactive protein in patients with major depressive disorder. Brain Behav Immun. 2015 Oct;49:206-15.

6 Stuart MJ, Baune BT. Chemokines and chemokine receptors in mood disorders, schizophrenia, and cognitive impairment: a systematic review of biomarker studies. Neurosci Biobehav Rev. 2014 May;42:93-115.

7 Reaux-Le Goazigo A, Van Steenwinckel J, Rostene W, Melik Parsadaniantz S. Current status of chemokines in the adult CNS. Prog Neurobiol. 2013 May;104:67-92.

8 Eyre HA, Air T, Pradhan A, Johnston J, Lavretsky $\mathrm{H}$, Stuart MJ, et al. A meta-analysis of chemokines in major depression. Prog Neuropsychopharmacol Biol Psychiatry. 2016 Jul 4;68:1-8.

9 Milenkovic VM, Sarubin N, Hilbert S, Baghai TC, Stöffler F, Lima-Ojeda JM, et al. Macrophage-derived chemokine: a putative marker of pharmacological therapy response in major depression? Neuroimmunomodulation. 2017; 24(2):106-12.
10 Yoshie O. Role of chemokines in trafficking of lymphocytes and dendritic cells. Int J Hematol. 2000 Dec;72(4):399-407.

11 Köhler-Forsberg O, Lydholm CN, Hjorthøj C, Nordentoft M, Mors O, Benros ME. Efficacy of anti-inflammatory treatment on major depressive disorder or depressive symptoms: meta-analysis of clinical trials. Acta Psychiatr Scand. 2019 May; 139(5): 404-19.

12 Zhou M, Greenhill S, Huang S, Silva TK, Sano $\mathrm{Y}, \mathrm{Wu}$ S, et al. CCR5 is a suppressor for cortical plasticity and hippocampal learning and memory. Elife. 2016;5:e20985.

13 Gates TM, Cysique LA, Siefried KJ, Chaganti J, Moffat KJ, Brew BJ. Maravirocintensified combined antiretroviral therapy improves cognition in virally suppressed HIV-associated neurocognitive disorder. AIDS. 2016 Feb 20;30(4):591600 .
Association of CCR5 and CCL5 with

MDD and Cognitive Impairment
Neuroimmunomodulation 2020;27:152-162 DOI: $10.1159 / 000513093$ 
14 Samson M, Labbe O, Mollereau C, Vassart G, Parmentier M. Molecular cloning and functional expression of a new human CC-chemokine receptor gene. Biochemistry. 1996 Mar 19; 35(11):3362-7.

15 de Oliveira CE, Oda JM, Losi Guembarovski R, de Oliveira KB, Ariza CB, Neto JS, et al. CC chemokine receptor 5: the interface of host immunity and cancer. Dis Markers. 2014;2014:126954.

16 Ward SG, Westwick J. Chemokines: understanding their role in T-lymphocyte biology. Biochem J. 1998 Aug 1;333(Pt 3):457-70.

17 Murphy PM. Viral exploitation and subversion of the immune system through chemokine mimicry. Nat Immunol. 2001 Feb;2(2):116-22.

18 Brelot A, Chakrabarti LA. CCR5 revisited: how mechanisms of HIV entry govern AIDS pathogenesis. J Mol Biol. 2018 Aug 17;430(17):255789.

19 Hirako IC, Assis PA, Galvão-Filho B, Luster AD, Antonelli LR, Gazzinelli RT. Monocyte-derived dendritic cells in malaria. Curr Opin Microbiol. 2019 Dec;52:139-50.

20 Velasco-Velázquez M, Xolalpa W, Pestell RG. The potential to target CCL5/CCR5 in breast cancer. Expert Opin Ther Targets. 2014 Nov 18(11):1265-75.

21 Aldinucci D, Colombatti A. The inflammatory chemokine CCL5 and cancer progression. Mediators Inflamm. 2014;2014:292376.

22 Joy MT, Ben Assayag E, Shabashov-Stone D, Liraz-Zaltsman S, Mazzitelli J, Arenas M, et al. CCR5 is a therapeutic target for recovery after stroke and traumatic brain injury. Cell. 2019 Feb 21;176(5):1143-e13.

23 Mueller A, Strange PG. CCL3, acting via the chemokine receptor CCR5, leads to independent activation of Janus kinase 2 (JAK2) and Gi proteins. FEBS Lett. 2004 Jan;570(1-3):126-2.

24 Navratilova Z. Polymorphisms in CCL2 \& CCL5 chemokines/chemokine receptors genes and their association with diseases. Biomed Pap Med Fac Univ Palacky Olomouc Czech Repub. 2006 Nov; 150(2):191-204.

25 Vangelista L, Secchi M, Lusso P. Rational design of novel HIV-1 entry inhibitors by RANTES engineering. Vaccine. 2008 Jun 6;26(24):3008-15.

26 Marques RE, Guabiraba R, Russo RC, Teixeira MM. Targeting CCL5 in inflammation. Expert Opin Ther Targets. 2013 Dec;17(12):1439-60.

27 Ogłodek EA, Szota A, Just MJ, Moś D, Araszkiewicz A. Comparison of chemokines (CCL- 5 and SDF-1), chemokine receptors (CCR-5 and CXCR-4) and IL-6 levels in patients with different severities of depression. Pharmacol Rep. 2014 Oct;66(5):920-6.

28 Hamilton M. A rating scale for depression. Neurol Neurosurg Psychiatry. 1960 Feb;23(1): 56-62.

29 Wu MJ, Passos IC, Bauer IE, Lavagnino L, Cao $B$, Zunta-Soares GB, et al. Individualized identification of euthymic bipolar disorder using the Cambridge Neuropsychological Test Automated Battery (CANTAB) and machine learning. J Affect Disord. 2016 Mar 1;192:219-25.
30 Sweeney JA, Kmiec JA, Kupfer DJ. Neuropsychologic impairments in bipolar and unipolar mood disorders on the CANTAB neurocognitive battery. Biol Psychiatry. 2000 Oct 1;48(7): 674-84.

31 Association AP. Diagnostic and statistical man ual of mental disorders. 4th ed. American Psychiatric Press; 1994.

32 Team RC. R: a language and environment for statistical computing. R Foundation for Statistical Computing; 2013.

33 Bates D, Mächler M, Bolker B, Walker S. Fitting linear mixed-effects models Usinglme4. J Stat Soft. 2015;67(1):1-48.

34 Kuznetsova A, Brockhoff PB, Christensen RHB. lmerTest: Tests for random and fixed effects for linear mixed effect models (lmer objects of lme4 package).; 2016.

35 Hilbert S, Stadler M, Lindl A, Naumann F, Bühner M. Analyzing longitudinal intervention studies with linear mixed models. Test Psychometry Methodol Appl Psychol. 2019;26: 101-19.

36 Barbosa IG, Rocha NP, Vieira EL, Camkurt MA, Huguet RB, Guimarães FTL, et al. Decreased percentage of CD4+ lymphocytes expressing chemokine receptors in bipolar disorder. Acta Neuropsychiatr. 2019 Oct;31(5):24651.

37 Mindt S, Neumaier M, Hoyer C, Sartorius A, Kranaster L. Cytokine-mediated cellular immune activation in electroconvulsive therapy: a CSF study in patients with treatment-resistant depression. World J Biol Psychiatry. 2020 Feb; 21(2):139-47.

38 Grassi-Oliveira R, Brieztke E, Teixeira A, Pezzi JC, Zanini M, Lopes RP, et al. Peripheral chemokine levels in women with recurrent major depression with suicidal ideation. Rev Bras Psiquiatr. 2012 Mar;34(1):71-5.

39 Leighton SP, Nerurkar L, Krishnadas R, Johnman C, Graham GJ, Cavanagh J. Chemokines in depression in health and in inflammatory illness: a systematic review and meta-analysis. Mol Psychiatry. 2018 Jan;23(1):48-58.

40 Chen X, Hu Q, Zhang K, Teng H, Li M, Li D, et al. The clock-controlled chemokine contributes to neuroinflammation-induced depression. FASEB J. 2020 Jun;34(6):8357-66.

41 Dahl J, Ormstad H, Aass HC, Malt UF, Bendz LT, Sandvik L, et al. The plasma levels of various cytokines are increased during ongoing depression and are reduced to normal levels after recovery. Psychoneuroendocrinology. 2014 Jul; 45:77-86.

42 Greeson JM, Gettes DR, Spitsin S, Dubé B, Benton TD, Lynch KG, et al. The selective serotonin reuptake inhibitor citalopram decreases human immunodeficiency virus receptor and coreceptor expression in immune cells. Biol Psychiatry. 2016 Jul 1;80(1):33-9.

43 Di Prisco S, Merega E, Lanfranco M, Casazza S, Uccelli A, Pittaluga A. Acute desipramine restores presynaptic cortical defects in murine experimental autoimmune encephalomyelitis by suppressing central CCL5 overproduction. Br J Pharmacol. 2014 May;171(9):2457-67.
44 Vollmar P, Nessler S, Kalluri SR, Hartung HP, Hemmer B. The antidepressant venlafaxine ameliorates murine experimental autoimmune encephalomyelitis by suppression of pro-inflammatory cytokines. Int J Neuropsychopharmacol. 2009 May;12(4):525-36.

45 Roumestan C, Michel A, Bichon F, Portet K, Detoc M, Henriquet C, et al. Anti-inflammatory properties of desipramine and fluoxetine. Respir Res. 2007 May 3;8(1):35.

46 Haapakoski R, Mathieu J, Ebmeier KP, Alenius $\mathrm{H}$, Kivimäki M. Cumulative meta-analysis of interleukins 6 and $1 \beta$, tumour necrosis factor $\alpha$ and $\mathrm{C}$-reactive protein in patients with major depressive disorder. Brain Behav Immun. 2015 Oct;49:206-15.

47 Köhler CA, Freitas TH, Maes M, de Andrade NQ, Liu CS, Fernandes BS, et al. Peripheral cytokine and chemokine alterations in depression: a meta-analysis of 82 studies. Acta Psychiatr Scand. 2017 May;135(5):373-87.

48 Köhler CA, Freitas TH, Stubbs B, Maes M, Sol$\mathrm{mi}$ M, Veronese N, et al. Peripheral alterations in cytokine and chemokine levels after antidepressant drug treatment for major depressive disorder: systematic review and meta-analysis. Mol Neurobiol. 2018;55(5):4195-206.

49 Więdłocha M, Marcinowicz P, Krupa R, Janoska-Jaździk M, Janus M, Dębowska W, et al. Effect of antidepressant treatment on peripheral inflammation markers: a meta-analysis. Prog Neuropsychopharmacol Biol Psychiatry. 2018 Jan 3;80(Pt C):217-26.

50 Liu JJ, Wei YB, Strawbridge R, Bao Y, Chang S Shi L, et al. Peripheral cytokine levels and response to antidepressant treatment in depression: a systematic review and meta-analysis. Mol Psychiatry. 2020 Feb;25(2):339-50.

51 Allers K, Schneider T. CCR5 $\Delta 32$ mutation and HIV infection: basis for curative HIV therapy. Curr Opin Virol. 2015 Oct;14:24-9.

52 Muxel SM, Borelli SD, Amarante MK, VoltarelliJC, Aoki MN, de Oliveira CE, et al. Association study of CCR5 delta 32 polymorphism among the HLA-DRB1 Caucasian population in Northern Paraná, Brazil. J Clin Lab Anal. 2008; 22(4):229-33.

53 Karam W, Jurjus R, Khoury N, Khansa H, Assad C, Zalloua P, et al. Frequency of the CCR5-delta 32 chemokine receptor gene mutation in the Lebanese population. East Mediterr Health J. 2004 Jul-Sep;10(4-5):671-5.

54 Egerházi A, Balla P, Ritzl A, Varga Z, Frecska E, Berecz R. Automated Neuropsychological Test Battery in depression: preliminary data. Neuropsychopharmacol Hung. 2013 Mar;15(1):5-11.

55 Semkovska M, Quinlivan L, O’Grady T, Johnson R, Collins A, O'Connor J, et al. Cognitive function following a major depressive episode: a systematic review and meta-analysis. Lancet Psychiatry. 2019 Oct;6(10):851-61.

56 Ozcan S, Gica S, Gulec H. Suicidal behavior in treatment resistant major depressive disorder patients treated with transmagnetic stimulation (TMS) and its relationship with cognitive functions. Psychiatry Res. 2020 Feb 19;286:112873. 